\title{
THE EFFECT OF REDUCED INTEGRATION IN THE STEKLOV EIGENVALUE PROBLEM*
}

\author{
María G. Armentano ${ }^{1}$
}

\begin{abstract}
In this paper we analyze the effect of introducing a numerical integration in the piecewise linear finite element approximation of the Steklov eigenvalue problem. We obtain optimal order error estimates for the eigenfunctions when this numerical integration is used and we prove that, for singular eigenfunctions, the eigenvalues obtained using this reduced integration are better approximations than those obtained using exact integration when the mesh size is small enough.
\end{abstract}

Mathematics Subject Classification. 65D30, 65N25, 65N30.

Received: December 20, 2002. Revised: September 3, 2003.

\section{INTRODUCTION}

The aim of this paper is to analyze the effect of introducing a numerical integration in the piecewise linear finite element approximation of the Steklov eigenvalue problem.

Increasing attention has recently been paid to the problem of approximating the vibration modes of a structure in contact with an incompressible fluid. The most usual procedure in engineering practice is to eliminate the fluid variable by using the so-called added mass formulation [5,9]. This consists of taking into account the effect of the fluid by means of a Neumann-to-Dirichlet operator on the fluid-solid interface. This approach yields an eigenvalue problem similar to the Steklov eigenvalue problem considered here.

There are relatively few papers treating the effect of numerical integration on eigenvalue approximation. For second order selfadjoint eigenvalue problems Banerjee and Osborn [3] prove that finite element approximations with quadrature rules satisfy the same estimates that hold with exact integration when the quadrature rules have appropriate degrees of precision. In [1], Armentano and Durán analyze the effect of a quadrature rule known as "mass-lumping" for second order selfadjoint eigenvalue problems.

For a Steklov eigenvalue problem optimal order error estimates in $H^{1}$ norm are obtained in [5] for the piecewise linear finite element approximation when exact integration is used. As far as we know, error estimates for the eigenfunction have not been proved when some quadrature rule is introduced.

The goal of this paper is to obtain optimal error estimates in $H^{1}$ and $L^{2}$-norms for the eigenfunctions when a quadrature rule is introduced in the computation of the right-hand side of the weak form of the equation of the Steklov eigenvalue problem. In order to obtain these estimates we prove that the order of the difference between the eigenfunction approximation obtained using the quadrature rule and the eigenfunction approximation with

\footnotetext{
Keywords and phrases. Finite elements, Steklov eigenvalue problem, reduced integration.

* Supported by ANPCyT under grant PICT 03-05009 and by CONICET under grant PIP 0660/98.

1 Departamento de Matemática, Facultad de Ciencias Exactas y Naturales, Universidad de Buenos Aires, 1428 Buenos Aires, Argentina. e-mail: garmenta@dm.uba.ar
} 
exact integration is higher than the order between the eigenfunction approximation with exact integration and the exact one.

Moreover, using our error estimates we will show that, for singular eigenfunctions, the eigenvalues obtained using reduced integration are better approximations that those obtained using exact integration when the mesh size is small enough. So, we extend the results obtained in [1] for the second order selfadjoint eigenvalue problems to the Steklov eigenvalue problem.

The paper is organized as follows. First, in Section 2, we present the Steklov eigenvalue problem and the approximation problem with and without quadrature rule. Section 3 deals with error estimates in $H^{1}$ and $L^{2}$-norms for the eigenfunctions. Finally in Section 4 we use the error estimates obtained in Section 3 to prove that, for singular eigenfunctions, the eigenvalues obtained using the reduced integration introduced in Section 2 are better approximations that those obtained using exact integration when the mesh size is small enough.

\section{The Steklov eigenvalue problem}

Let $\Omega \subset \mathbb{R}^{2}$ be a bounded polygonal domain. We consider the following Steklov eigenvalue problem [2]:

$$
\begin{aligned}
-\triangle u+u & =0 & \text { in } \Omega \\
\frac{\partial u}{\partial n} & =\lambda u & \text { on } \Gamma=\partial \Omega .
\end{aligned}
$$

The variational problem associated with (2.1) is given by:

Find $\lambda$ and $u \in H^{1}(\Omega), u \neq 0$ satisfying

$$
\begin{aligned}
a(u, v) & =\lambda \int_{\Gamma} u v \quad \forall v \in H^{1}(\Omega) \\
\|u\|_{L^{2}(\Gamma)} & =1
\end{aligned}
$$

where $a(u, v)=\int_{\Omega} \nabla u \nabla v+\int_{\Omega} u v$, which is continuous and coercive on $H^{1}(\Omega)$.

It is known that the solution of this problem is given by a sequence of pairs $\left(\lambda_{j}, u_{j}\right)$, with positive eigenvalues $\lambda_{j}$ diverging to $+\infty$ [10]. We assume the eigenvalues to be increasingly ordered: $0<\lambda_{1} \leq \cdots \leq \lambda_{j} \leq \cdots$ The associated eigenfunctions satisfy $u_{j} \in H^{1+r}(\Omega)$, where $r=1$ if $\Omega$ is convex and $r<\frac{\pi}{\omega}$ (with $\omega$ being the largest inner angle of $\Omega$ ) otherwise (see for example [8]).

In order to approximate the eigenvalue $\lambda$ and its associated eigenfunction $u$ we consider $\left\{\mathcal{T}_{h}\right\}$ a triangulation of $\Omega$ such that any two triangles in $\mathcal{T}_{h}$ share at most a vertex or an edge. Let $h$ stand for the mesh-size; namely $h=\max _{T \in \mathcal{T}_{h}} h_{T}$, with $h_{T}$ being the diameter of the triangle $T$. We assume that the family of triangulations $\mathcal{T}_{h}$ satisfies a minimal angle condition, i.e., there exists a constant $\sigma>0$ such that $\frac{h_{T}}{\rho_{T}} \leq \sigma$, where $\rho_{T}$ is the diameter of the largest circle contained in $T$.

We consider the standard finite element space:

$$
V_{h}=\left\{v \in H^{1}(\Omega):\left.v\right|_{T} \in \mathcal{P}_{1} \quad \forall T \in \mathcal{T}_{h}\right\}
$$

where $\mathcal{P}_{1}$ denotes the space of linear polynomials.

Then, the standard finite element approximation problem is the following:

Find $\lambda_{h}$ and $u_{h} \in V_{h}, u_{h} \neq 0$ such that

$$
\begin{aligned}
a\left(u_{h}, v\right) & =\lambda_{h} \int_{\Gamma} u_{h} v \quad \forall v \in V_{h} \\
\left\|u_{h}\right\|_{L^{2}(\Gamma)} & =1 .
\end{aligned}
$$

Let $\beta_{j}, 1 \leq j \leq N_{h}\left(N_{h}=\right.$ number of nodes $)$ be the Lagrange basis of degree one, i.e., $\left.\beta_{j}\right|_{T} \in \mathcal{P}_{1} \quad \forall T \in \mathcal{T}_{h}$ such that $\beta_{j}\left(n_{i}\right)=\delta_{i, j}$ where $n_{i}$ denotes the node $i$. The generalized eigenvalue problem is given by: find $u_{h}=\sum_{j=1}^{N_{h}} z_{j} \beta_{j}$ such that

$$
A z=\lambda_{h} B z
$$


where

and

$$
A_{i, j}=\int_{\Omega} \nabla \beta_{i} \nabla \beta_{j}+\int_{\Omega} \beta_{i} \beta_{j}
$$

$$
B_{i, j}=\int_{\Gamma} \beta_{i} \beta_{j}
$$

Another possible discretization is obtained by using quadrature rule on the right hand side of (2.3). If we use in the computation of the matrix $B$ the trapezoid rule we obtain another generalized eigenvalue problem

$$
A z=\lambda_{h}^{I} \tilde{B} z
$$

where the matrix $\tilde{B}$ is diagonal. The variational problem is in this case:

Find $\lambda_{h}^{I}$ and $u_{h}^{I} \in V_{h}, u_{h}^{I} \neq 0$ such that

$$
\begin{aligned}
a\left(u_{h}^{I}, v\right) & =\lambda_{h}^{I} \int_{\Gamma} I_{h}\left(u_{h}^{I} v\right) \quad \forall v \in V_{h}, \\
\left\|u_{h}^{I}\right\|_{L^{2}(\Gamma)} & =1
\end{aligned}
$$

where $I_{h}$ denotes the piecewise linear interpolation on the vertices of the triangulation $\mathcal{T}_{h}$ which lies on $\Gamma$.

The two problems given above reduce to generalized eigenvalue problems where the matrix $A$ is positive definite and symmetric, and the matrices $B$ and $\tilde{B}$ are non-negative definite, symmetric, and both of them have rank $M_{h}=$ number of vertices on $\Gamma$. They attain a finite number of eigenpairs $\left(\lambda_{h, j}, u_{h, j}\right)$ and $\left(\lambda_{h, j}^{I}, u_{h, j}^{I}\right), 1 \leq$ $j \leq M_{h}$, respectively, with positive eigenvalues which we assume increasingly ordered: $0<\lambda_{h, 1} \leq \cdots \leq \lambda_{h, M_{h}}$ and $0<\lambda_{h, 1}^{I} \leq \cdots \leq \lambda_{h, M_{h}}^{I}$.

In order to simplify notation from now on we will drop the subindex $j$ in $\lambda_{j}, \lambda_{h, j}, \lambda_{h, j}^{I}, u_{j}, u_{h, j}, u_{h, j}^{I}$ and we denote by $C$ a generic constant not necessarily the same at each occurrence.

\section{Error estimates}

Following the arguments given in [5] error estimates in $H^{1}$ norm can be obtained for the eigenfunction approximation using the standard finite element method (2.3), i.e., it can be seen that there exists a constant $C$ such that

$$
\left\|u-u_{h}\right\|_{H^{1}(\Omega)} \leq C h^{r} .
$$

In order to obtain error estimates for the eigenfunction, when a quadrature rule is introduced, we will use the spectral approximation theory given in [2].

Let $T, T_{h}: H^{1}(\Omega) \rightarrow H^{1}(\Omega)$ be the bounded linear operators defined by

$$
\begin{aligned}
& \left\{\begin{array}{l}
T f \in H^{1}(\Omega) \\
a(T f, v)=b(f, v) \forall v \in H^{1}(\Omega)
\end{array}\right. \\
& \left\{\begin{array}{l}
T_{h} f \in V_{h} \\
a\left(T_{h} f, v\right)=b(f, v) \forall v \in V_{h}
\end{array}\right.
\end{aligned}
$$

where $b(f, v)=\int_{\Gamma} f v$. The non-zero eigenvalues of $T$ are the reciprocals of the eigenvalues of $(2.2)$ and the non-zero eigenvalues of $T_{h}$ are the reciprocals of the eigenvalues of (2.3) and $T$ and (2.2), $T_{h}$ and (2.3) have the same eigenfunctions. Now, we introduce another operator $T_{h}^{I}: H^{1}(\Omega) \rightarrow H^{1}(\Omega)$ defined by

$$
\left\{\begin{array}{l}
T_{h}^{I} f \in V_{h} \\
a\left(T_{h}^{I} f, v\right)=b^{I}(f, v) \forall v \in V_{h}
\end{array}\right.
$$


where $b^{I}(f, v)=\int_{\Gamma} I\left(P_{h}(f) v\right)$ with $P_{h}$ being the $L^{2}$ projection on $\Gamma$ onto the piecewise linear continuous functions, i.e.,

$$
\int_{\Gamma} P_{h}(f) v=\int_{\Gamma} f v \quad \forall v \in V_{h} .
$$

It is easy to see that the non-zero eigenvalues of $T_{h}^{I}$ are the reciprocals of those of (2.4) and $T_{h}^{I}$ and (2.4) have the same eigenfunctions.

First, we will obtain error estimates in $L^{2}(\Gamma)$ for the eigenfunction approximation when exact integration is used. The following estimate holds

Proposition 3.1. Let $(\lambda, u)$ and $\left(\lambda_{h}, u_{h}\right)$ be the solutions of problems (2.2) and (2.3), respectively. Then there exists a constant $C$ such that

$$
\left\|u_{h}-u\right\|_{L^{2}(\Gamma)} \leq C h^{\frac{3}{2} r} .
$$

Proof. Let $e=T f-T_{h} f$ with $f$ being an eigenfunction of the problem (2.2). In order to obtain error estimates in $L^{2}(\Gamma)$ we consider the following auxiliary problem:

$$
\begin{aligned}
-\triangle \phi+\phi & =0 & & \text { in } \Omega \\
\frac{\partial \phi}{\partial n} & =e & & \text { on } \Gamma .
\end{aligned}
$$

Let $\phi_{I} \in V_{h}$ be the Lagrange interpolation of $\phi$. By subtracting (3.7) from (3.6) we have that $a\left(e, \phi_{I}\right)=0$. Then,

$$
\begin{aligned}
\|e\|_{L^{2}(\Gamma)}^{2} & =\int_{\Gamma} e^{2}=\int_{\Gamma} \frac{\partial \phi}{\partial n} e=\int_{\Omega} \nabla \phi \nabla e+\int_{\Omega} \phi e \\
& =a(\phi, e)=a\left(\phi-\phi_{I}, e\right) \leq\left\|\phi-\phi_{I}\right\|_{H^{1}(\Omega)}\|e\|_{H^{1}(\Omega)} .
\end{aligned}
$$

Using standard finite element estimates [6,7] and the same arguments given in Proposition 4.4 of [5] we have that

$$
\|e\|_{L^{2}(\Gamma)}^{2} \leq C h^{\frac{r}{2}}\|\phi\|_{H^{1+\frac{r}{2}(\Omega)}}\|e\|_{H^{1}(\Omega)} \leq C h^{\frac{r}{2}}\|e\|_{L^{2}(\Gamma)}\|e\|_{H^{1}(\Omega)} .
$$

Therefore by using the error estimates in $H^{1}$ (see [5]) we have that

$$
\|e\|_{L^{2}(\Gamma)}^{2} \leq C h^{\frac{3}{2} r}\|e\|_{L^{2}(\Gamma)}\|f\|_{H^{1}(\Omega)}
$$

but, since $f$ is an eigenfunction we have that there exists a constant $C$ such that $\|f\|_{H^{1}(\Omega)} \leq C\|f\|_{L^{2}(\Gamma)}$ and so,

$$
\|e\|_{L^{2}(\Gamma)} \leq C h^{\frac{3}{2} r}\|f\|_{L^{2}(\Gamma)}
$$

and using the spectral approximation theory (see [2]) we obtain the desired result.

For second order-elliptic eigenvalue problems, reference [3] contains error estimates considering numerical integration under the assumption that the eigenfunction is smooth. However, their arguments can be used to obtain error estimates when the eigenfunction are non-smooth. As far as we know, error estimates for the Steklov eigenvalue problem have not been proved when some integration rule is introduced. Our next goal is to obtain error estimates for the Steklov eigenvalue problem using the reduced integration defined in (2.4). We will prove that the order of the difference between the eigenfunction approximation obtained using the quadrature rule and the eigenfunction approximation with exact integration is higher than the order between the eigenfunction approximation with exact integration and the exact one. 
The following approximation result holds:

Theorem 3.1. There exists a constant $C>0$ such that for any $f \in H^{1}(\Omega)$ we have

$$
\left\|\left(T_{h}-T_{h}^{I}\right) f\right\|_{H^{1}(\Omega)} \leq C h\|f\|_{H^{1}(\Omega)}
$$

Moreover, if $f$ is an eigenfunction of the problem (2.2), we have

$$
\left\|\left(T_{h}-T_{h}^{I}\right) f\right\|_{H^{1}(\Omega)} \leq C h^{\frac{3}{2}}\|f\|_{H^{1}(\Omega)} .
$$

Proof. For any $f \in H^{1}(\Omega)$ and $v \in V_{h}$ from (3.7)-(3.9) we have that

$$
a\left(\left(T_{h}-T_{h}^{I}\right) f, v\right)=\int_{\Gamma} f v-I\left(P_{h}(f) v\right)=\int_{\Gamma} P_{h}(f) v-I\left(P_{h}(f) v\right)
$$

Let $\left\{\ell_{k}\right\}_{1 \leq k \leq M_{h}}$ be the edges of the mesh lying on $\Gamma$ then,

$$
\int_{\Gamma} P_{h}(f) v-I\left(P_{h}(f) v\right)=\sum_{\ell_{k} \subset \Gamma} \int_{\ell_{k}} P_{h}(f) v-I\left(P_{h}(f) v\right) \leq \sum_{\ell_{k} \subset \Gamma}\left\|P_{h}(f) v-I\left(P_{h}(f) v\right)\right\|_{L^{1}\left(\ell_{k}\right)} .
$$

Using standard error estimates for interpolation (see for example [7]), the fact that $P_{h}(f)$ and $v$ are functions in $V_{h}$ and Cauchy-Schwarz inequality we have

$$
\sum_{\ell_{k} \subset \Gamma}\left\|P_{h}(f) v-I\left(P_{h}(f) v\right)\right\|_{L^{1}\left(\ell_{k}\right)} \leq C \sum_{\ell_{k} \subset \Gamma}\left|\ell_{k}\right|^{2}\left|P_{h}(f) v\right|_{W^{2,1}\left(\ell_{k}\right)} \leq C \sum_{\ell_{k} \subset \Gamma}\left|\ell_{k}\right|^{2}\left\|\frac{\partial P_{h}(f)}{\partial \ell_{k}}\right\|_{L^{2}\left(\ell_{k}\right)}\left\|\frac{\partial v}{\partial \ell_{k}}\right\|_{L^{2}\left(\ell_{k}\right)}
$$

Now using the following inverse estimate: $\left\|\frac{\partial w}{\partial \ell_{k}}\right\|_{L^{2}\left(\ell_{k}\right)} \leq \frac{C}{\left|\ell_{k}\right|^{\frac{1}{2}}}\|w\|_{H^{\frac{1}{2}\left(\ell_{k}\right)}} \forall w \in V_{h}[6,7]$ and Cauchy-Schwarz inequality we get

$$
\int_{\Gamma} P_{h}(f) v-I\left(P_{h}(f) v\right) \leq C h \sum_{\ell_{k} \subset \Gamma}\left\|P_{h}(f)\right\|_{H^{\frac{1}{2}\left(\ell_{k}\right)}}\|v\|_{H^{\frac{1}{2}\left(\ell_{k}\right)}} \leq C h\left(\sum_{\ell_{k} \subset \Gamma}\left\|P_{h}(f)\right\|_{H^{\frac{1}{2}}\left(\ell_{k}\right)}^{2}\right)^{\frac{1}{2}}\left(\sum_{\ell_{k} \subset \Gamma}\|v\|_{H^{\frac{1}{2}}\left(\ell_{k}\right)}^{2}\right)^{\frac{1}{2}} .
$$

Since $\sum_{\ell_{k} \subset \Gamma}\|w\|_{H^{\frac{1}{2}\left(\ell_{k}\right)}}^{2} \leq C\|w\|_{H^{\frac{1}{2}(\Gamma)}}^{2}$ for any $w \in H^{\frac{1}{2}}(\Gamma)$ we have

$$
\int_{\Gamma} P_{h}(f) v-I\left(P_{h}(f) v\right) \leq C h\left\|P_{h}(f)\right\|_{H^{\frac{1}{2}}(\Gamma)}\|v\|_{H^{\frac{1}{2}}(\Gamma)}
$$

Then using that there exits a constant $C$ such that $\left\|P_{h}(f)\right\|_{H^{\frac{1}{2}}(\Gamma)} \leq C\|f\|_{H^{\frac{1}{2}(\Gamma)}}([6],[7])$ and a trace theorem we get

$$
\int_{\Gamma} P_{h}(f) v-I\left(P_{h}(f) v\right) \leq C h\|f\|_{H^{\frac{1}{2}(\Gamma)}}\|v\|_{H^{\frac{1}{2}(\Gamma)}} \leq C h\|f\|_{H^{1}(\Omega)}\|v\|_{H^{1}(\Omega)} .
$$

So, the first result follows by taking $v=T_{h} f-T_{h}^{I} f$.

If $f$ is an eigenfunction of the problem $(2.2) f$ lies in $H^{1+r}(\Omega)$ with $\frac{1}{2}<r \leq 1$. 
So, instead of (3.12) we have in this case

$$
\begin{aligned}
\int_{\Gamma} P_{h}(f) v-I\left(P_{h}(f) v\right) & \leq C \sum_{\ell_{k} \subset \Gamma}\left|\ell_{k}\right|^{2}\left\|\frac{\partial P_{h}(f)}{\partial \ell_{k}}\right\|_{L^{2}\left(\ell_{k}\right)}\left\|\frac{\partial v}{\partial \ell_{k}}\right\|_{L^{2}\left(\ell_{k}\right)} \leq C h^{\frac{3}{2}} \sum_{\ell_{k} \subset \Gamma}\left\|P_{h}(f)\right\|_{H^{1}\left(\ell_{k}\right)}\|v\|_{H^{\frac{1}{2}}\left(\ell_{k}\right)} \\
& \leq C h^{\frac{3}{2}}\left(\sum_{\ell_{k} \subset \Gamma}\left\|P_{h}(f)\right\|_{H^{1}\left(\ell_{k}\right)}^{2}\right)^{\frac{1}{2}}\left(\sum_{\ell_{k} \subset \Gamma}\|v\|_{H^{\frac{1}{2}}\left(\ell_{k}\right)}^{2}\right)^{\frac{1}{2}}
\end{aligned}
$$

and using the same arguments given above we have

$$
\begin{aligned}
\int_{\Gamma} P_{h}(f) v-I\left(P_{h}(f) v\right) & \leq C h^{\frac{3}{2}}\left\|P_{h}(f)\right\|_{H^{1}(\Gamma)}\|v\|_{H^{\frac{1}{2}}(\Gamma)} \leq C h^{\frac{3}{2}}\|f\|_{H^{1}(\Gamma)}\|v\|_{H^{\frac{1}{2}(\Gamma)}} \\
& \leq C h^{\frac{3}{2}}\|f\|_{H^{1+r}(\Omega)}\|v\|_{H^{\frac{1}{2}(\Gamma)}} \leq C h^{\frac{3}{2}}\|f\|_{H^{1+r}(\Omega)}\|v\|_{H^{1}(\Omega)} .
\end{aligned}
$$

Since $f$ is an eigenfunction from the same arguments given in Proposition 4.4 in [5] and the trace theorem we know that there exists a constant $C$ such that $\|f\|_{H^{1+r}(\Omega)} \leq C\|f\|_{H^{\frac{1}{2}(\Gamma)}} \leq C\|f\|_{H^{1}(\Omega)}$. Therefore, the proof concludes by taking $v=T_{h} f-T_{h}^{I} f$.

Corollary 3.1. Let $u$ and $u_{h}^{I}$ be the eigenfunctions of the problems (2.2) and (2.4) respectively. There exists a constant $C$ such that

$$
\left\|u-u_{h}^{I}\right\|_{H^{1}(\Omega)} \leq C h^{r} .
$$

Proof. It is a consequence of the error estimate $\left\|\left(T-T_{h}\right) f\right\|_{H^{1}(\Omega)} \leq C h^{r}\|f\|_{H^{1}(\Omega)}$ [5], Theorem 3.1 and Theorem 7.1 in $[2]$.

In order to obtain $L^{2}(\Gamma)$ error estimates for eigenfunctions when reduced integration is used we consider the following theorem.

Theorem 3.2. Let $f$ be the eigenfunction of the problem (2.2). There exists a constant $C>0$ such that

$$
\left\|\left(T_{h}-T_{h}^{I}\right) f\right\|_{L^{2}(\Gamma)} \leq C h^{\frac{3}{2}}\|f\|_{L^{2}(\Gamma)} .
$$

Moreover, if $\left\{\mathcal{T}_{h}\right\}$ is quasi-uniform, i.e., there exists a constant $\delta>0$ such that $\frac{h}{h_{T}} \leq \delta, \forall T \in \mathcal{T}_{h}$, then, for any $\gamma \in\left(0, \frac{1}{2}\right), \gamma \leq \frac{r}{2}$ there exists a constant $C=C(\gamma)$ such that

$$
\left\|\left(T_{h}-T_{h}^{I}\right) f\right\|_{L^{2}(\Gamma)} \leq C h^{\frac{3}{2}+\gamma}\|f\|_{L^{2}(\Gamma)} .
$$

In particular, if $r<1$ we have $\left\|\left(T_{h}-T_{h}^{I}\right) f\right\|_{L^{2}(\Gamma)} \leq C h^{\frac{3}{2}+\frac{r}{2}}\|f\|_{L^{2}(\Gamma)}$.

Proof. Let $e=T_{h} f-T_{h}^{I} f$ with $f$ being an eigenfunction of the problem (2.2) and $\phi$ being the solution of the auxiliary problem

$$
\begin{aligned}
-\triangle \phi+\phi & =0 & & \text { in } \Omega \\
\frac{\partial \phi}{\partial n} & =e & & \text { on } \Gamma .
\end{aligned}
$$

The variational problem associated with (3.15) is given by

$$
a(\phi, v)=\int_{\Gamma} e v \quad \forall v \in H^{1}(\Omega) .
$$


Let $\phi_{h} \in V_{h}$ be the solution of the discrete variational problem

$$
a\left(\phi_{h}, v\right)=\int_{\Gamma} e v \quad \forall v \in V_{h} .
$$

Then,

$$
\begin{aligned}
\|e\|_{L^{2}(\Gamma)}^{2} & =a\left(e, \phi_{h}\right)=a\left(T_{h} f-T_{h}^{I} f, \phi_{h}\right)=\int_{\Gamma} f \phi_{h}-I\left(P_{h}(f) \phi_{h}\right) \\
& =\int_{\Gamma} P_{h}(f) \phi_{h}-I\left(P_{h}(f) \phi_{h}\right) .
\end{aligned}
$$

Then,

$$
\begin{aligned}
\int_{\Gamma} P_{h}(f) \phi_{h}-I\left(P_{h}(f) \phi_{h}\right) & \leq \sum_{\ell_{k} \subset \Gamma}\left\|P_{h}(f) \phi_{h}-I\left(P_{h}(f) \phi_{h}\right)\right\|_{L^{1}\left(\ell_{k}\right)} \leq C \sum_{\ell_{k} \subset \Gamma}\left|\ell_{k}\right|^{2}\left|P_{h}(f) \phi_{h}\right|_{W^{2,1}\left(\ell_{k}\right)} \\
& \leq C \sum_{\ell_{k} \subset \Gamma}\left|\ell_{k}\right|^{2}\left\|\frac{\partial P_{h}(f)}{\partial \ell_{k}}\right\|_{L^{2}\left(\ell_{k}\right)}\left\|\frac{\partial \phi_{h}}{\partial \ell_{k}}\right\|_{L^{2}\left(\ell_{k}\right)}
\end{aligned}
$$

By the same arguments used in the proof of Theorem 3.1 we have

$$
\left\|P_{h}(f) \phi_{h}-I\left(P_{h}(f) \phi_{h}\right)\right\|_{L^{1}(\Gamma)} \leq C h^{\frac{3}{2}}\left\|P_{h}(f)\right\|_{H^{1}(\Gamma)}\left\|\phi_{h}\right\|_{H^{\frac{1}{2}}(\Gamma)} \leq C h^{\frac{3}{2}}\|f\|_{H^{1+r}(\Omega)}\left\|\phi_{h}\right\|_{H^{1}(\Omega)} .
$$

Since $\left\|\phi_{h}\right\|_{H^{1}(\Omega)} \leq C\|e\|_{L^{2}(\Gamma)}$ and $\|f\|_{H^{1+r}(\Omega)} \leq C\|f\|_{H^{\frac{1}{2}(\Gamma)}} \leq C\|f\|_{H^{1}(\Omega)} \leq C\|f\|_{L^{2}(\Gamma)}$ we have that

$$
\left\|T_{h} f-T_{h}^{I} f\right\|_{L^{2}(\Gamma)} \leq C h^{\frac{3}{2}}\|f\|_{L^{2}(\Gamma)}
$$

and the first result holds.

Now, we assume that the triangulation $\left\{\mathcal{T}_{h}\right\}$ satisfies the quasi-uniform condition, i.e., there is a constant $\delta>0$ such that $\frac{h}{h_{T}} \leq \delta, \forall T \in \mathcal{T}_{h}$.

Let $\gamma \in\left(0, \frac{1}{2}\right), \gamma \leq \frac{r}{2}$ then, using the inverse estimate $\left\|\frac{\partial \phi_{h}}{\partial \ell_{k}}\right\|_{L^{2}\left(\ell_{k}\right)} \leq \frac{C}{\left|\ell_{k}\right|^{\frac{1}{2}-\gamma}}\left\|\phi_{h}\right\|_{H^{\frac{1}{2}+\gamma}\left(\ell_{k}\right)}$ and Cauchy-Schwarz inequality, instead of (3.17) we have in this case

$$
\begin{aligned}
\left\|P_{h}(f) \phi_{h}-I\left(P_{h}(f) \phi_{h}\right)\right\|_{L^{1}(\Gamma)} & \leq C h^{\frac{3}{2}+\gamma}\left\|P_{h}(f)\right\|_{H^{1}(\Gamma)}\left\|\phi_{h}\right\|_{H^{\frac{1}{2}+\gamma}(\Gamma)} \leq C h^{\frac{3}{2}+\gamma}\|f\|_{H^{1+r}(\Omega)}\left\|\phi_{h}\right\|_{H^{\frac{1}{2}+\gamma}(\Gamma)} \\
& \leq C h^{\frac{3}{2}+\gamma}\|f\|_{H^{1+r}(\Omega)}\left\|\phi_{h}\right\|_{H^{1+\gamma}(\Omega)} .
\end{aligned}
$$

Let $\Pi_{h}$ be the Lagrange interpolation operator, from Theorem 2.6 in [4] we know that there exists a constant $C$ such that

$$
\left\|\phi-\Pi_{h} \phi\right\|_{H^{1+\gamma}(\Omega)} \leq C\|\phi\|_{H^{1+\gamma}(\Omega)}
$$

and therefore

$$
\left\|\Pi_{h} \phi\right\|_{H^{1+\gamma}(\Omega)} \leq C\|\phi\|_{H^{1+\gamma}(\Omega)} .
$$

On the other hand from the inverse inequality given in Theorem 2.9 in [4] and standard error estimates we have that

$$
\left\|\phi_{h}-\Pi_{h} \phi\right\|_{H^{1+\gamma}(\Omega)} \leq C \frac{1}{h^{\gamma}}\left\|\phi_{h}-\Pi_{h} \phi\right\|_{H^{1}(\Omega)} \leq C\|\phi\|_{H^{1+\gamma}(\Omega)} .
$$

So, $\left\|\phi_{h}\right\|_{H^{1+\gamma}(\Omega)} \leq\left\|\phi_{h}-\Pi_{h} \phi_{h}\right\|_{H^{1+\gamma}(\Omega)}+\left\|\Pi_{h} \phi\right\|_{H^{1+\gamma}(\Omega)} \leq C\|\phi\|_{H^{1+\gamma}(\Omega)}$. 
Since $\|\phi\|_{H^{1+\gamma}(\Omega)} \leq C\|\phi\|_{H^{1+\frac{r}{2}(\Omega)}} \leq C\|e\|_{L^{2}(\Gamma)}$ and $\|f\|_{H^{1+r}(\Omega)} \leq C\|f\|_{L^{2}(\Gamma)}$ we conclude that

$$
\left\|T_{h} f-T_{h}^{I} f\right\|_{L^{2}(\Gamma)} \leq C h^{\frac{3}{2}+\gamma}\|f\|_{L^{2}(\Gamma)}
$$

and we complete the proof.

Now, we have the $L^{2}(\Gamma)$ error estimates for eigenfunctions when reduced integration is used.

Corollary 3.2. Let $(\lambda, u)$ and $\left(\lambda_{h}^{I}, u_{h}^{I}\right)$ be the solutions of problems (2.2) and (2.4) respectively. Then there exists a constant $C$ such that

$$
\left\|u-u_{h}^{I}\right\|_{L^{2}(\Gamma)} \leq C h^{\frac{3}{2} r} .
$$

Proof. The result is a consequence of (3.10), Theorem 3.2 and the spectral approximation theory [2].

\section{Advantages of Reduced integration}

The goal of this section is to show that if the eigenfunction of problem (2.2) is singular the eigenvalue approximation given by using reduced integration (2.4) is better than the eigenvalue approximation given by the standard finite element (2.3) for $h$ small enough. So, we extend the results obtained in [1] for second order-elliptic eigenvalue problems to the Steklov eigenvalue problem.

First, we will show that the eigenvalue obtained by reduced integration is always below the one obtained by the standard finite element approximation, i.e, $\lambda_{h}^{I} \leq \lambda_{h}$.

For each boundary edge $\left\{\ell_{k}\right\}_{1 \leq k \leq M_{h}}$ we denote by $p_{1}\left(\ell_{k}\right)$ and $p_{2}\left(\ell_{k}\right)$ the extremes of the edge $\ell_{k}$. Then we have

Lemma 4.1. For any $v_{h} \in V_{h}$,

$$
\int_{\Gamma}\left(I_{h}\left(v_{h}^{2}\right)-v_{h}^{2}\right)=\frac{1}{6} \sum_{\ell_{k} \subset \Gamma}\left(v_{h}\left(p_{1}\left(\ell_{k}\right)\right)-v_{h}\left(p_{2}\left(\ell_{k}\right)\right)\right)^{2}\left|\ell_{k}\right|
$$

in particular

$$
\int_{\Gamma} I_{h}\left(v_{h}^{2}\right) \geq \int_{\Gamma} v_{h}^{2}
$$

Proof. Since $v_{h}$ is a piecewise linear function we observe that

$$
\int_{\Gamma} v_{h}^{2}=\sum_{\ell_{k} \subset \Gamma}\left(v_{h}^{2}\left(p_{1}\left(\ell_{k}\right)\right)+v_{h}^{2}\left(p_{2}\left(\ell_{k}\right)\right)+4 v_{h}^{2}\left(m_{\ell_{k}}\right)\right) \frac{\left|\ell_{k}\right|}{6}
$$

where $m_{\ell_{k}}$ denote the midpoint of the edge $\ell_{k}$. From $v_{h}\left(m_{\ell_{k}}\right)=\frac{v_{h}\left(p_{1}\left(\ell_{k}\right)\right)+v_{h}\left(p_{2}\left(\ell_{k}\right)\right)}{2}$ and

$$
\int_{\Gamma} I_{h}\left(v_{h}^{2}\right)=\sum_{\ell_{k} \subset \Gamma}\left(v_{h}^{2}\left(p_{1}\left(\ell_{k}\right)\right)+v_{h}^{2}\left(p_{2}\left(\ell_{k}\right)\right)\right) \frac{\left|\ell_{k}\right|}{2}
$$

we have that

$$
\begin{aligned}
\int_{\Gamma}\left(I\left(v_{h}^{2}\right)-v_{h}^{2}\right) & =\sum_{\ell_{k} \subset \Gamma}\left(v_{h}^{2}\left(p_{1}\left(\ell_{k}\right)\right)+v_{h}^{2}\left(p_{2}\left(\ell_{k}\right)\right)\right) \frac{\left|\ell_{k}\right|}{2}-\left(v_{h}^{2}\left(p_{1}\left(\ell_{k}\right)\right)+v_{h}^{2}\left(p_{2}\left(\ell_{k}\right)\right)+v_{h}\left(p_{1}\left(\ell_{k}\right) v_{h}\left(p_{2}\left(\ell_{k}\right)\right)\right) \frac{\left|\ell_{k}\right|}{3}\right. \\
& =\sum_{\ell_{k} \subset \Gamma}\left(v_{h}^{2}\left(p_{1}\left(\ell_{k}\right)\right)+v_{h}^{2}\left(p_{2}\left(\ell_{k}\right)\right)-2 v_{h}\left(p_{1}\left(\ell_{k}\right)\right) v_{h}\left(p_{2}(\ell)\right)\right) \frac{\left|\ell_{k}\right|}{6}
\end{aligned}
$$

and therefore the Lemma holds. 
As a consequence of the previous Lemma we have that

Corollary 4.1. For any $v_{h} \in V_{h}, \gamma \in\left(0, \frac{1}{2}\right)$, there exists a constant $C=C(\gamma)$ such that

$$
0 \leq \int_{\Gamma} I_{h}\left(v_{h}^{2}\right)-v_{h}^{2} \leq C h^{1+2 \gamma}\left\|v_{h}\right\|_{H^{\frac{1}{2}+\gamma}(\Gamma)}^{2}
$$

Proof. Using Lemma 4.1 and the fact that $\frac{\partial v_{h}}{\partial \ell_{k}}=\frac{v_{h}\left(p_{2}\left(\ell_{k}\right)\right)-v_{h}\left(p_{1}\left(\ell_{k}\right)\right)}{\left|\ell_{k}\right|}$ we have that

$$
\int_{\Gamma}\left(I_{h}\left(v_{h}^{2}\right)-v_{h}^{2}\right)=\frac{1}{6} \sum_{\ell_{k} \subset \Gamma}\left(\left(v_{h}\left(p_{1}\left(\ell_{k}\right)\right)-v_{h}\left(p_{2}\left(\ell_{k}\right)\right)\right)^{2}\left|\ell_{k}\right|=\frac{1}{6} \sum_{\ell_{k} \subset \Gamma}\left(\frac{\partial v_{h}}{\partial \ell_{k}}\right)^{2}\left|\ell_{k}\right|^{3}=\frac{1}{6} \sum_{\ell_{k} \subset \Gamma}\left\|\frac{\partial v_{h}}{\partial \ell_{k}}\right\|_{L^{2}\left(\ell_{k}\right)}^{2}\left|\ell_{k}\right|^{2} .\right.
$$

Since, for any $\gamma \in\left(0, \frac{1}{2}\right)$, there exists a constant $C=C(\gamma)$ such that $\left\|\frac{\partial v_{h}}{\partial \ell_{k}}\right\|_{L^{2}\left(\ell_{k}\right)} \leq \frac{C}{\left|\ell_{k}\right|^{\frac{1}{2}-\gamma}}\left\|v_{h}\right\|_{H^{\frac{1}{2}+\gamma}\left(\ell_{k}\right)}$ we obtain

$$
\int_{\Gamma} I_{h}\left(v_{h}^{2}\right)-v_{h}^{2} \leq \frac{1}{6} C \sum_{\ell_{k} \subset \Gamma}\left\|v_{h}\right\|_{H^{\frac{1}{2}+\gamma}\left(\ell_{k}\right)}^{2}\left|\ell_{k}\right|^{1+2 \gamma} \leq C h^{1+2 \gamma}\left\|v_{h}\right\|_{H^{\frac{1}{2}+\gamma}(\Gamma)}^{2}
$$

and we conclude the proof.

Theorem 4.1. Let $\lambda, \lambda_{h}$ and $\lambda_{h}^{I}$, be the eigenvalues of problems (2.2)-(2.4) respectively. Then

$$
\lambda \leq \lambda_{h} \quad \text { and } \quad \lambda_{h}^{I} \leq \lambda_{h}
$$

Proof. Let $T, T_{h}$ and $T_{h}^{I}$ be the operators defined in (3.6)-(3.8) and let $\mu_{j}, \mu_{h, j}$ and $\mu_{h, j}^{I}$, the corresponding eigenvalues which we assume non increasingly ordered, i.e., $\mu_{1} \geq \mu_{2} \geq \cdots \mu_{j} \geq \cdots, \mu_{h, 1} \geq \mu_{h, 2} \geq \cdots \geq \mu_{h, M_{h}}$ and $\mu_{h, 1}^{I} \geq \mu_{h, 2}^{I} \geq \cdots \geq \mu_{h, M_{h}}^{I}$.

It is known that the eigenvalues can be characterized using the maximum-minimum principle [10], i.e., for any $j, 1 \leq j \leq M_{h}$ we have that

and

$$
\mu_{j}=\max _{V_{j}} \min _{v \in V_{j}} \frac{b(v, v)}{a(v, v)}
$$

$$
\mu_{h, j}=\max _{V_{h, j}} \min _{v_{h} \in V_{h, j}} \frac{b\left(v_{h}, v_{h}\right)}{a\left(v_{h}, v_{h}\right)} \quad \mu_{h, j}^{I}=\max _{V_{h, j}} \min _{v_{h} \in V_{h, j}} \frac{b^{I}\left(v_{h}, v_{h}\right)}{a\left(v_{h}, v_{h}\right)}
$$

where $V_{j}$ denote any subspace of $H^{1}(\Omega)$ of dimension $j$ and $V_{h, j}$ denote any subspace of $V_{h}$ of dimension $j$.

Since $V_{h} \subset H^{1}(\Omega)$ we have that $\mu_{j} \geq \mu_{h, j}$.

In view of Lemma 4.1 we have that for any $j, 1 \leq j \leq M_{h}$

$$
\frac{b^{I}\left(v_{h}, v_{h}\right)}{a\left(v_{h}, v_{h}\right)} \geq \frac{b\left(v_{h}, v_{h}\right)}{a\left(v_{h}, v_{h}\right)} \quad \forall v_{h} \in V_{h, j}
$$

So, $\mu_{h, j}^{I} \geq \mu_{h, j}, 1 \leq j \leq M_{h}$.

The proof concludes by using that the non-zero eigenvalues of $T, T_{h}$ and $T_{h}^{I}$ are the reciprocals of the eigenvalues of (2.2), (2.3) and (2.4), respectively.

The next Lemma, which follows from Lemma 5.1 of [3] or Lemma 2.2 of [1], gives an expression for the difference between $\lambda$ and the approximation given by reduced integration $\lambda_{h}^{I}$.

Lemma 4.2. Let $(\lambda, u)$ and $\left(\lambda_{h}^{I}, u_{h}^{I}\right)$ be the solutions of problems (2.2) and (2.4) respectively. Then we have that

$$
\lambda_{h}^{I}-\lambda=\left\|u_{h}^{I}-u\right\|_{H^{1}(\Omega)}^{2}-\lambda\left\|u_{h}^{I}-u\right\|_{L^{2}(\Gamma)}^{2}-\lambda_{h}^{I}\left(\int_{\Gamma} I_{h}\left(\left(u_{h}^{I}\right)^{2}\right)-\left(u_{h}^{I}\right)^{2}\right) .
$$


Now, we assume that the triangulation $\left\{\mathcal{T}_{h}\right\}$ satisfies the quasi-uniform condition, i.e., there is a constant $\delta>0$ such that $\frac{h}{h_{T}} \leq \delta, \forall T \in \mathcal{T}_{h}$. Then we have the following result.

Corollary 4.2. Let $\lambda$ and $\lambda_{h}^{I}$ be the eigenvalues of problems (2.2) and (2.4) respectively. If there exists a constant $C$ such that $\left\|u_{h}^{I}-u\right\|_{H^{1}(\Omega)} \geq C h^{r}$ with $r<1$ then, for $h$ small enough, we have

$$
\lambda \leq \lambda_{h}^{I}
$$

Proof. The proof follows the same argument given in [1]. From Lemma 4.2 we know that

$$
\lambda_{h}^{I}-\lambda=\left\|u_{h}^{I}-u\right\|_{H^{1}(\Omega)}^{2}-\lambda\left\|u_{h}^{I}-u\right\|_{L^{2}(\Gamma)}^{2}-\lambda_{h}^{I}\left(\int_{\Gamma} I_{h}\left(\left(u_{h}^{I}\right)^{2}\right)-\left(u_{h}^{I}\right)^{2}\right) .
$$

From Corollary 4.1, taking $\gamma=\frac{r}{2}$ we have

$$
\int_{\Gamma} I_{h}\left(\left(u_{h}^{I}\right)^{2}\right)-\left(u_{h}^{I}\right)^{2} \leq C h^{1+r}\left\|u_{h}^{I}\right\|_{H^{\frac{1}{2}+\frac{r}{2}}(\Gamma)}^{2}
$$

Since, $\left\|u_{h}^{I}\right\|_{H^{\frac{1}{2}+\frac{r}{2}(\Gamma)}} \leq C\left\|u_{h}^{I}\right\|_{H^{1+\frac{r}{2}}(\Omega)} \leq C\left(\left\|u_{h}^{I}-\Pi_{h} u\right\|_{H^{1+\frac{r}{2}}(\Omega)}+\left\|\Pi_{h} u\right\|_{H^{1+\frac{r}{2}}(\Omega)}\right)$ using Theorems 2.6 and 2.9 in [4], Corollary 3.1 and standard error estimates for interpolation we obtain

$$
\left\|u_{h}^{I}\right\|_{H^{1+\frac{r}{2}}(\Omega)} \leq C\left(\frac{1}{h^{\frac{r}{2}}}\left\|u_{h}^{I}-\Pi_{h} u\right\|_{H^{1}(\Omega)}+\|u\|_{H^{1+\frac{r}{2}}(\Omega)}\right) \leq C
$$

So, the order of the third term on the right hand side of (4.24) is $h^{1+r}$.

From our hypothesis, the first term on the right hand side of (4.24) is greater than a constant times $h^{2 r}$ and, in view of Corollary 3.2, the order of the second term is $h^{3 r}$. Therefore if $h$ is small enough, the sign of $\lambda_{h}^{I}-\lambda$ is given by the first term on (4.24) so, we conclude the proof.

Acknowledgements. The author wishes to thank Prof. Rodolfo Rodriguez for his multiple and useful suggestions.

\section{REFERENCES}

[1] M.G. Armentano and R.G. Durán, Mass lumping or not mass lumping for eigenvalue problems. Numer. Methods Partial Differential Equations 19 (2003) 653-664.

[2] I. Babuska and J. Osborn, Eigenvalue Problems, Handbook of Numerical Analysis, Vol. II. Finite Element Methods (Part. 1) (1991).

[3] U. Banerjee and J. Osborn, Estimation of the effect of numerical integration in finite element eigenvalue approximation. Numer. Math. 56 (1990) 735-762.

[4] F.B. Belgacem and S.C. Brenner, Some nonstandard finite element estimates with applications to 3D Poisson and Signorini problems. Electron. Trans. Numer. Anal. 12 (2001) 134-148.

[5] A. Bermudez, R. Rodriguez and D. Santamarina, A finite element solution of an added mass formulation for coupled fluid-solid vibrations. Numer. Math. 87 (2000) 201-227.

[6] S.C. Brenner and L.R. Scott, The Mathematical Theory of Finite Element Methods. Springer-Verlag, New York (1994).

[7] P. Ciarlet, The Finite Element Method for Elliptic Problems. North-Holland, Amsterdam (1978).

[8] P. Grisvard, Elliptic Problems in Nonsmooth Domain. Pitman Boston (1985).

[9] H.J.-P. Morand and R. Ohayon, Interactions Fluids-Structures. Rech. Math. Appl. 23 (1985).

[10] H.F. Weinberger, Variational Methods for Eigenvalue Approximation. SIAM, Philadelphia (1974). 